\title{
Cause, Pattern and Outcome of Fractures Occurring in the Pediatric Population of Rural Tertiary Health Care Center of Nepal
}

\author{
Karki DB, ${ }^{1}$ Rokaya PK, ${ }^{1}$ Rawal M, ${ }^{1}$ Bhandari PB, ${ }^{1}$ Limbu D, ${ }^{1}$ Shrestha $S,{ }^{2}$ Acharya BD, ${ }^{1}$ Thapa SD ${ }^{1}$ \\ ${ }^{1}$ Department of Orthopaedics, Karnali Academy of Health Sciences, Jumla, Nepal, ${ }^{2}$ Department of \\ Pediatrics, Karnali Academy of Health Sciences, Jumla, Nepal.
}

Corresponding Author:

Dr. Dhan Bahadur Karki

Karnali Academy of Health Sciences, Jumla, Nepal

Email: dhankarki017@gmail.com

\begin{abstract}
Introduction: Pediatric trauma is a significant burden to health care worldwide. In Karnali province, most of the cases present late. The aim of this study is to determine demographic characteristics, cause, pattern, treatment and outcome of pediatric fractures in tertiary health care center in Jumla.

Methods: This is a retrospective study done in patients younger than 16 years who presented to our hospital between January 2016 to December 2017. We analyzed demographic data, injury characteristics, fracture pattern, time of presentation, treatment modalities and outcome.

Results: There were total of 1028 patients out of which $60 \%$ were males. Mean duration of presentation to hospital was 4.8 days. Most common mechanism of injury was fall from height. $85.60 \%$ were managed conservatively and $14.40 \%$ were managed operatively. Postoperative outcome was good in $71 \%$ of the cases, fair in $28 \%$ and poor in $1 \%$ cases. Forearm fracture was the most common presentation, accounting for 358 cases (34.82\%) followed by Supracondylar humerus fracture accounting $23.34 \%$.

Conclusions: Pediatric fractures constitute a huge proportion of cases in our hospital. As pediatric bones unite readily, the treatment outcome is good in most of the cases even after conservative management and after delayed presentation.
\end{abstract}

Keywords: conservative management; delayed presentation; pediatric fractures; supracondylar fractures.

\section{INTRODUCTION}

The incidence of pediatric fracture is increasing; approximately $50 \%$ of all children will fracture at least one bone during childhood. The increasing incidence in children has been mainly attributed to increased sports participation. Skeletal trauma accounts for $10-15 \%$ of all childhood injuries, with approximately $15-30 \%$ of this representing physeal injuries. ${ }^{1}$

Pediatric trauma is a significant burden to health care world wide. ${ }^{2}$ Knowledge regarding epidemiology, fracture pattern and outcome of fracture helps to plan to reduce the burden to community and healthcare. ${ }^{3}$ Injuries represent a frequent cause of admission into pediatric orthopedic and traumatology departments. Fractures occur in about $25 \%$ of all injured children. ${ }^{4}$ The mechanisms of these injuries vary from accidental falls during recreational activities to road traffic accidents. Non-operative management is the mainstay of treatment of pediatric fractures, with reported good outcomes owing to the active remodelling potential of children's periosteum which speeds up the fracture healing process. ${ }^{5,6}$ Forearm fractures are most common long bones fractures in children, occurring with annual incidence of approximately 1.5 per hundred children per year 
comprising up to $40 \%$ of all pediatric fracture. ${ }^{7}$

Supracondylar fracture of the humerus is one of the most talked about and often encountered injury (only after clavicle and both bone forearm fracture) in pediatric age group with a male predominance accounting for $16 \%$ of all pediatric fractures and $60 \%$ of all pediatric elbow fractures, classically occurring as a result of fall on an outstretched hand. ${ }^{8-10}$

The management of pediatric fractures in low income countries is suboptimal, predisposing injured children to increased risk of carrying disabilities and other complications into adulthood. ${ }^{3}$ The aim of this study is to determine demographic characteristics, cause, pattern, treatment and outcome of pediatric fractures in tertiary health care center in Jumla.

\section{METHODS}

A retrospective analysis of 1028 cases between birth and 16 years of age managed in Karnali academy of health science between January 2016 and December 2017 was done. All fractures occurring between birth and 16 years of age were analyzed. From the data collected, we analyzed demographic data, duration of injury and presentation, fracture characteristics, mechanism of injury, type of treatment and outcome of treatment at one year of follow up. The outcome was evaluated as poor if there was mal-union that couldn't be corrected by bone remodeling; acceptable when insufficient reduction was corrected by bone remodeling without resultant functional impairment and good when reduction was anatomical. ${ }^{11}$ Ethical consideration was approved from Nepal Health Research Council (NHRC) prior to study. Data was analyzed using SPSS v 22.

\section{RESULTS}

Pediatric fractures comprised $36.5 \%$ of all fracture cases presenting to our hospital during the time interval of our study. There were total of 1028 cases. Among them, there was male predominance with $60.01 \%$ (617 cases). Most common age group was 5-8 years of age in girls and 6-9 years in boys. Mean age of presentation was 6.3 years in girls and 7.6 years in boys. Mean duration of presentation to hospital was 4.8 days (range $=1-67$ days). Spiral fractures $30 \%$, transverse $28 \%$ oblique fractures $22 \%$, comminuted fractures $14 \%$ and others $6 \%$.

Most of the fractures were on the right limb 60\% except for supracondylar humerus fracture which was more common on the left side.

Forearm fracture was the most common presentation, accounting for $34.82 \%$ (358 cases). Supracondylar humerus fracture accounted for $23.34 \%$ (240 cases). Other fractures were shaft of femur $11.67 \%$ (120 cases), tibia fracture $9.72 \%$ (100 cases), lateral condyle humerus fracture $3.4 \%$ (35 cases), clavicle fracture 2.91\%(30 cases), elbow dislocation $0.97 \%$ (10 cases), shaft of humerus fracture $2.43 \%$ (25 cases), medial condyle of humerus fracture $0.97 \%$ (10 cases), proximal humerus fracture $1.94 \%$ (20 cases), hand injuries $6.80 \%$ (70 cases), spine fractures $0.6 \%$ ( 7 cases) and neck of femur $0.29 \%$ (3 cases)

\begin{tabular}{|l|l|}
\hline Table 1. & Number \\
\hline Fractures & 358 \\
\hline Forearm & 240 \\
\hline Supracondylar humerus & 120 \\
\hline Shaft of femur & 100 \\
\hline Shaft of tibia & 35 \\
\hline Lateral condyle humerus & 10 \\
\hline Elbow dislocation & 25 \\
\hline Shaft of humerus & 30 \\
\hline Clavicle & 10 \\
\hline Medial condyle of humerus & 20 \\
\hline Proximal humerus & 70 \\
\hline Hand injuries & 7 \\
\hline Spine & 3 \\
\hline Neck of femur &
\end{tabular}

Most common mechanism of injury was fall from height (45\%). $40 \%$ of the cases were due to sports injury 


\section{Mechanism of injury}

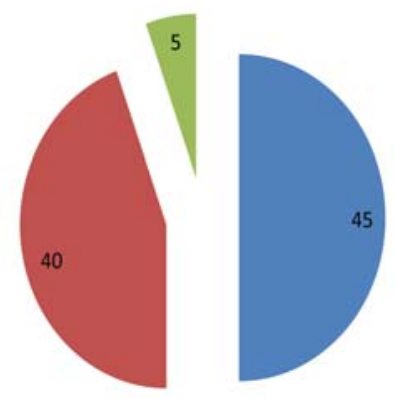

Uall from height

asports

in others

\section{Figure 1.}

Among them, 14,4\% were managed operatively and the remaining $85.60 \%$ (578 cases) were managed conservatively. Outcome was good in 71 $\%$ of the cases, fair in $28 \%$ and poor in $1 \%$ cases.

\section{DISCUSSION}

Pediatric fractures constitute a major proportion of cases in our hospital and the incidence is increasing every year. Being a rural area, late presentation and neglected cases are common in this region. Many disabilities of adulthood have their root in childhood injuries. Lack of adequate health facilities, specialists \& education in public and poverty lead to delayed presentation, suboptimal treatment and disabilities. Fracture of any extremity is rarely life-threatening, the unique anatomy and the intimatelocation of neurovascular structures in elbow often result in a spectrum of injuries with associated complications. ${ }^{12,13}$

Epidemiological studies have suggested that $18 \%$ of children will experience a fracture by the age of nine years with children between the ages of 5 and 14 having the highest incidence. The risk of sustaining a fracture between birth and 16 years of age is reported to be between 42-64\% for boys and between $27-40 \%$ for girls. This further suggests the importance of studies for childhood fractures. There are few literatures regarding pediatric fractures from different parts of the world. But research is limited in our country regarding the same. Working in a rural tertiary health center in a poor community area like Jumla, there are many challenges in managing childhood fractures. The mean duration of presentation was
5.8 days for patients of Jumla and 4.5 days for patients of other districts. Lack of transportation and poverty was the main cause behind this. Fractures were more common among boys as they are more out-going and active in sports. Although most fractures in children heal well without longterm complications, certain fractures, especially those involving the physis and articular surfaces, have the potential to cause significant morbidity.

Most of the fractures were managed conservatively. Forearm fractures were managed with closed reduction and cast application under anesthesia. 70 cases required operative management due to failure of closed reduction and 20 cases required open reduction. Similarly, type I and II supracondylar fractures were managed conservatively while type III fractures underwent operative management with closed reduction and percutaneous pinning. 20 cases required open reduction. All cases of lateral condyle humerus fracture were managed with open reduction. Similarly, all cases of neck of femur fracture were managed operatively with percutaneous pinning. Shaft of femur fracture was managed with hip spica cast in children younger than 5 years and with flexible nail in older children. Plating was done in 2 cases. Tibial fractures were managed conservatively with closed reduction and cast application in 20 cases, flexible nail in 18 cases and plating in 2 cases. Humerus fractures were managed conservatively with u-slab and hanging cast. Proximal humerus fractures were also managed conservatively. Clavicle fracture was managed conservatively with clavicular brace and arm pouch sling. Spine fracture was managed with Knight Taylor brace and bed rest. Medial condyle humerus fracture was managed conservatively with above elbow posterior slab.

Outcome of pediatric fracture was good in majority of the cases even with conservative management. This suggests that children, unlike adults, can remodel fractures as they grow, especially those in the plane of motion of the adjacent joint. Nonunion in children is rare and was not encountered in our study. 


\section{CONCLUSIONS}

Pediatric fractures are a common occurrence in our hospital. Most of the fractures unite readily following both conservative and operative management. Delay presentation to the hospital is a major problem in the remote area.

\section{Conflict of Interest: None}

\section{REFERENCES}

1. K.A.Egol. Pediateric orthopedic surgery: General Principle. $5^{\text {th }}$ edition. Hand Book of Fracture 2015:509

2. World Health Organization. World Report on Child Injury Prevention: Summary. Geneva: WHO; 2008

3. Marc Leroy Guifo, Joel NoutakdieTochie, Blondel Nana Oumarou. Pediatric Fractures in sub-Saharan Tertiary care center: A cohort study analysis of demographic characteristic, clinical presentation, therapeutic patterns and outcomes. Pan African Medical Journal. 18/05/2017. 1-7

4. Heinrich Stephen, Drvaric David, Darr Kevin, MacEwen Dean G. The operative stabilization of pediatric diaphyseal Femur Fractures with Flexible Intramedullary Nails: a prospective analysis. Journal of PediatricOrthopaedics. 1994; 14(4): 501-7

5. Fractures de l'enfant monographies du grouped'étu deenorthopediepédiatrique. Montpellier Sauramps Medical. 2002; 213-221

6. NdomaNgatchoukpo V, Gaudeuille A, Journeau P. Traitementorthopédiques des fractures déplacés du quart inférieur du radius chez l'enfant. Rev Cames Sante.2014; 2(1): 9-13

7. Rockwood and Wilkins. Fracture in children.Chapter: fractures of distal radius and ulna. Eight edition. 489

8. Kasser JR, Beaty JH. Supracondylar fractures of the distal humerus. In: Rockwood and Wilkins' Fractures in Children, 5th, Beaty JH, Kasser JR. (Eds), Lippincott Williams \& Wilkins, Philadelphia 2001. Pp.577.
9. Villarin LA, Belk KE, Freid R. Emergency department evaluation and treatment [9] of elbow and forearm injuries. Emergency Medicine Clinics of North America. 1999;17(4):843-58

10. Campbell CC, Waters PM, Emans JB, Kasser JR, Millis MB. Neurovascular [10] injury and displacement in type III supracondylar humerus fractures. Journal of Pediatric Orthopaedics. 1995;15(1):47-52

11. Guifo ML,Tochie JN,Sosso MA.Paediatric fracture in sub-saharan tertiary care center:a cohort analysisof demographic characteristics,clinical presentation,theraputuc pattern and outcomes.Pan Afr Med J .2017 may18;27:46

12. Chai KK, Aik S, Sengupta S. Supracondylar fractures of the humerus in children-an epidemiological study of 132 consecutive cases. Med J Malaysia 2000;55(suppl C):39-43

13. Milbrandt TA, Copley LAB. Common elbow injuries in children: evaluation, treatment, and clinical outcomes. CurrOpinOrthop2004; 15:286-94 